\title{
Neural mechanisms of overt attention shifts to emotional faces
}

\author{
Louisa Kulke $\mathbf{a}^{\mathrm{a}, \mathrm{b}, *}$
}

a) Affective Neuroscience and Psychophysiology, University of Göttingen, Göttingen, Germany

b) Leibniz ScienceCampus Primate Cognition, Göttingen, Germany

* corresponding author, email: lkulke@uni-goettingen.de

Emotional faces draw attention and eye-movements towards them. However, the neural mechanisms of attention have mainly been investigated during fixation, which is uncommon in everyday life where people move their eyes to shift attention to faces. Therefore, the current study combined eye-tracking and Electroencephalography (EEG) to measure neural mechanisms of overt attention shifts to faces with happy, neutral and angry expressions, allowing participants to move their eyes freely towards the stimuli. Saccade latencies towards peripheral faces did not differ depending on expression and early neural response (P1) amplitudes and latencies were unaffected. However, the later occurring Early Posterior Negativity (EPN) was significantly larger for emotional than for neutral faces. This response occurs after saccades towards the faces. Therefore, emotion modulations only occurred after an overt shift of gaze towards the stimulus had already been completed. Visual saliency rather than emotional content may therefore drive early saccades, while later top-down processes reflect emotion processing.

\section{Keywords: Event-related brain potentials, Eye-tracking, Overt attention, Emotion, Faces}

\section{Highlights}

- Overt attention shifts towards peripheral emotional faces were investigated with combined EEG and eye-tracking

- Latency of eye-movements towards peripheral faces was unaffected by their emotional expression

- P1 was unaffected by emotional content in both amplitude and latency

- EPN amplitude was larger for emotional than neutral facial expressions

- Effects of facial expressions can successfully be investigated during overt attention shifts

\section{Introduction}

Emotional faces are both interesting and relevant to humans. From an evolutionary perspective, fast attention to emotional stimuli has an advantage, allowing humans to promptly react to danger, securing survival (Dickinson \& Dearing, 1979; see Lang \& Bradley, 2010 for a review). The attentional draw of emotional stimuli has been demonstrated in numerous studies. When emotional and neutral visual scenes are presented simultaneously in peripheral vision, participants have a higher probability of looking at the emotional picture first (Nummenmaa, Hyönä, \& Calvo, 2006) and reflexive saccades are faster towards emotional than neutral scenes (Nummenmaa, Hyönä, \& Calvo, 2009). Also, emotional faces cue attention towards their locations (Mogg \& Bradley, 1999; Pourtois, Grandjean, Sander, \& Vuilleumier, 2004), for example when they are displayed among neutral expressions in a visual search task (Schupp, Flaisch, Stockburger, \& Junghöfer, 2006). In everyday life, faces, particularly when viewed at a distance, attract gaze (Foulsham, Walker, \& Kingstone, 2011), suggesting that overt attention towards them plays a crucial role in guiding gaze. The emotional expressions that faces show may furthermore influence gaze patterns, but only few studies exist that study its effect on gaze shifting. Calvo and 
Nummenmaa (2008) suggested that participants orient faster towards happy than other facial expressions. Saccades towards peripherally presented faces were found to be faster when they displayed an emotional expression than a neutral one (Johnson Jr, 1988). In contrast, Hunt, Cooper, Hungr, and Kingstone (2007) found no difference in the latency of saccades to angry and happy or inverted faces in a visual search task. It therefore remains unclear, how eye-movements are affected by emotional faces.

On a neural level, emotional stimuli lead to increased responses compared to neutral stimuli (Vuilleumier, 2005; Vuilleumier, Armony, Driver, \& Dolan, 2001; Vuilleumier \& Huang, 2009). The increased neural responses can be measured with Electroencephalography (EEG), to determine Event-Related Potentials (ERPs), reflecting the time-course of stimulus processing with a high temporal accuracy. Emotional, compared to neutral, stimuli elicit an increased Early Posterior Negativity (EPN), a negative response occurring 250-300 ms after the onset of a stimulus in posterior areas of the brain (Hammerschmidt, Kulke, Broering, \& Schacht, 2018; Schupp, Junghöfer, Weike, \& Hamm, 2003). A later response, the Late Positive Potential (LPP, also LPC) has also been shown to be increased to happy (Bublatzky, Gerdes, White, Riemer, \& Alpers, 2014; Recio, Sommer, \& Schacht, 2011) and angry (Schupp et al., 2004) compared to neutral faces.

Some studies also found even earlier posterior responses to be modulated by emotional expressions of faces. In particular, fearful faces were found to affect the early posterior visual " $\mathrm{C} 1$ " response occurring after $60 \mathrm{~ms}$ (Pourtois et al., 2004). The amplitude of the early posterior positive "P1" response occurring around $100 \mathrm{~ms}$ also was shown to be modulated by emotional (Bublatzky et al., 2014), in particular angry (Rellecke, Sommer, \& Schacht, 2012) expressions and emotional compared to neutral words (Zhang et al., 2014). In contrast, other studies found no effects of emotion on the early $\mathrm{C} 1$ or $\mathrm{P} 1$, with effects occurring only on the facerelated "N170" component or later components (Batty \& Taylor, 2003; Leppänen, Moulson, Vogel-Farley, \& Nelson, 2007; Schupp et al., 2003; Zhang, Wang, Luo, \& Luo, 2012), although it is unclear whether effects on the N170 are related to emotional expressions (for a meta-analysis, see Lee et al., 2010) or to overlaps with the EPN (Rellecke et al., 2012). P1 latency was unaffected by emotional expressions when they were presented centrally (Batty \& Taylor, 2003). Therefore, it is still unclear whether very early attentional responses are affected by emotional content.

Previous studies mainly focused on measuring covert attention to stimuli, instructing participants to focus on one location and to inhibit any eye-movements. This methodology was chosen, as eyemovements can create artifacts in EEG data, distorting the measured responses (Corby \& Kopell, 1972; Croft \& Barry, 2000; Joyce, Gorodnitsky, \& Kutas, 2004; Luck, 2005). However, in everyday life attention shifts are often accompanied by an eyemovement; therefore, more natural overt shifts of attention need to be studied. This is particularly relevant as subtle differences exist between neural mechanisms of overt and covert attention shift, for example related to enhanced visual processing due to saccade planning (Saber, Pestilli, \& Curtis, 2015) and components reflecting saccade inhibition during covert attention shifts (Kulke, Atkinson, \& Braddick, 2016a). Technological advances make it possible to study neural mechanisms of attention during free viewing by combining eyetracking and EEG (Kulke et al., 2016a; Kulke, Atkinson, \& Braddick, 2016b). Kulke (2015) used a simple attention paradigm to demonstrate that latencies of saccades and the P1 were shorter when a salient peripheral target attracted undivided attention than when a competing target was decreasing attention to the peripheral target. Attention to physically salient targets is 
therefore already reflected in P1 latency during overt attention shifts.

However, neural mechanisms of attention to emotionally salient faces have not yet been investigated in an overt attention shift task. The aim of the current study was to combine EEG and eyetracking to study the neural mechanisms of overt attention shifts to emotional faces. It aimed to investigate whether latencies of saccades and early neural responses to peripheral face stimuli also differ as a function of emotional content, whether later ERPs also differ depending on emotional content. We expected saccade latencies and early posterior ERP latencies to differ between emotional and neutral faces. Furthermore, amplitudes of the posterior response $(\mathrm{P} 1)$, the EPN and the LPC were expected to be larger for emotional than neutral faces.

As people differ regarding their behavior, interactions with personality traits were further explored. Some people tend to avoid negative stimuli (behavioural inhibition system, BIS) or strive for positive stimuli (behavioural activation system, BAS) (Carver \& White, 1994). In the current task, we expected participants with high compared to low BIS scores to shift faster to angry faces, as they particularly attend to negative stimuli and should therefore react to them faster. Participants with high compared to low BAS scores were expected to shift faster to happy faces, as they particularly attend to positive stimuli. As participants who are better at recognizing emotional expressions may show larger behavioral and neural effects, interactions with the general ability to interpret facial emotions were measured using the "Reading Mind in the Eye" test (Baron-Cohen, Jolliffe, Mortimore, \& Robertson, 1997). Furthermore, the Autism Spectrum Quotient (AQ) (Baron-Cohen, Wheelwright, Skinner, Martin, \& Clubley,

\footnotetext{
${ }^{1}$ Note that a power analysis conducted in $\mathrm{G} *$ Power (Faul, Erdfelder, Lang, \& Buchner, 2007) software using the P1 effect sizes from Kulke (2015), a Power of 0.80 and alpha $=0.05$ determined that 20
}

2001) was used to control for clinical autistic traits.

\section{Method}

\section{Participants}

The study was pre-registered with the Open Science Framework (osf.io/vbk2e). Twenty-four healthy participants between 18 and 35 years (mean age $=23.33$ years, $S D=2.15)$ were tested, based on previous studies (Kulke et al. 2016; Hammerschmidt et al., 2017) ${ }^{1}$. No participant scored above the clinical cut-off of the AQ questionnaire. One additional participant needed to be excluded due to excessive noise in the EEG data. Despite sample size planning in the pre-registration, some effects were not significant. To exclude the possibility that this was due to low power, in a non-preregistered follow up study, 10 more participants were included (mean age $=$ 23.80 years, $\mathrm{SD}=1.6$ ) with further 2 excluded due to excessive noise in the eyetracking data (1) or not completing the full test session (1). The analysis of all 34 participants is reported in Supplement A. All participants scored below the clinical cut-off value of the AQ. Participants signed an informed consent before taking part and received course credit or monetary compensation (16€) in return for their participation, which took approximately 2 hours. The study was approved by the local ethics committee.

\section{Stimuli}

Thirty images of faces were selected from the Karolinska Directed Emotional Faces database (Calvo \& Nummenmaa, 2011) and luminance was controlled digitally using Adobe Photoshop CS6 software version 13.0.1 x64 and adjusted by measuring the mean luminance of stimuli and adjusting the value to be the same for all images, if required. Half of the images showed a female, the other half a male face. Ten

participants would provide sufficient power for a two-tailed test. The number of participants tested in this study is slightly higher to account for potential overestimation of the effects. 
respective images showed a happy, neutral and angry expression of the face of the same person. Images were presented on a liquid crystal display (LCD) screen at $80 \mathrm{~cm}$ distance from the participant. Each trial started with the presentation of a central fixation cross with a size of 50 pixel $(0.7 \mathrm{~cm}$, $0.5^{\circ}$ ) for a random inter-trial-interval between 1500 and $2500 \mathrm{~ms}$. Once participants fixated within 40 pixel $(0.56$ $\left.\mathrm{cm}, 0.4^{\circ}\right)$ around it for at least 150 samples $(300 \mathrm{~ms})$ at the end of or after the inter trial interval, a face appeared in the left or right periphery $\left(400\right.$ pixel $/ 5.6 \mathrm{~cm} / 4^{\circ}$ from the center, with a size of $324 \times 504$ pixel / $4.5 \times$ $7 \mathrm{~cm} / 3.4^{\circ} \times 5^{\circ}$ ) for at least $700 \mathrm{~ms}$. The side was counterbalanced for each image. Once participants fixated within 40 pixel $\left(0.56 \mathrm{~cm}, 0.4^{\circ}\right)$ of the image for at least 150 samples, an empty screen was presented. Subsequently, the next trial started. Stimuli were presented using Python and PsychoPy (Nummenmaa et al., 2009). An EyeLink 1000 eye-tracker recorded gaze at a rate of $500 \mathrm{~Hz}$ throughout each trial and raw eyetracking data was processed online and saved for later processing.

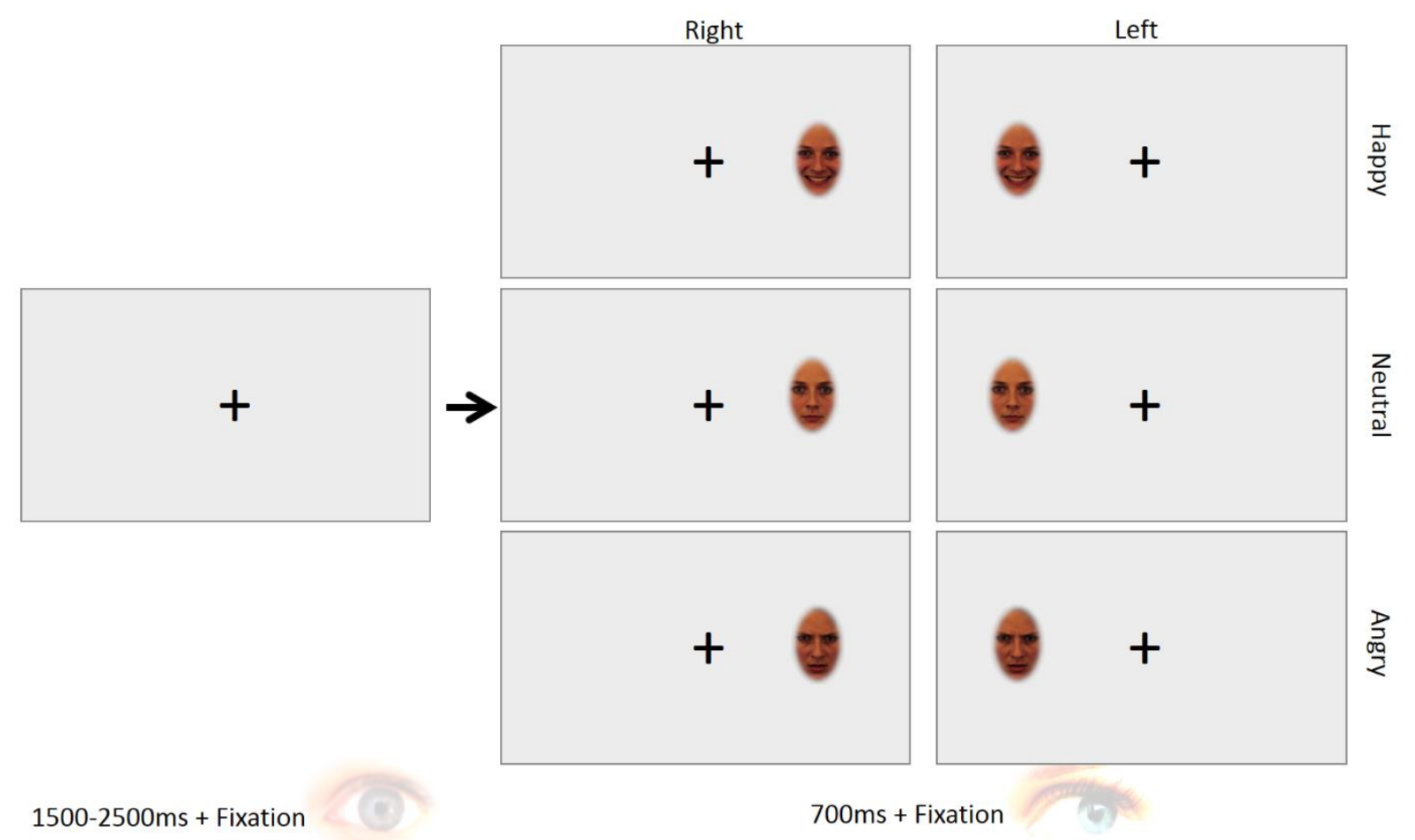

Figure 1. Schematic display of the screens presented during each trial.

\section{EEG}

EEG was recorded at a sampling rate of 512 $\mathrm{Hz}$ with a cap containing 64 active $\mathrm{Ag}-\mathrm{AgCl}$ electrodes, according to the extended international 10-20 system (Pivik et al., 1993). Scalp voltage signals were amplified by a BiosemiActiveTwo AD-Box, digitized with 24 bits. Electrode offsets were kept below a threshold of $+/-30 \mathrm{mV}$. Six external electrodes were applied below both eyes (vertical Electrooculography), to the outer canthi (horizontal Electrooculography) and to the left and right mastoids. Data was recorded with common mode sense (CMS) active electrode as reference, located between $\mathrm{PO} 3$ and $\mathrm{POz}$, and the driven right leg (DRL) passive electrode as ground electrode, located between $\mathrm{POz}$ and $\mathrm{PO} 4$.

\section{Procedure}

After providing informed consent, the EEG was applied and the eye-tracker was calibrated using a standard 9 point calibration and validation routine. Participants were asked to look at faces that appeared in the periphery. They completed 600 trials (200 per expression, 100 per side and expression, as in the study by Kulke et 
al. (2016a)) in randomized order, with short breaks after each block of 100 trials. After the study, participants completed the Autism Quotient Questionnaire (BaronCohen et al., 2001), the "Reading Mind in the Eye" test (Baron-Cohen et al., 1997) and the German version of the BIS/BAS test (Strobel, Beauducel, Debener, \& Brocke, 2001).

\section{Eye-tracking data processing}

Gaze positions were extracted and it was determined for each trial at what latency the first saccade towards the peripheral target appeared. Trials were excluded from further analysis if (1) the saccade was directed to the incorrect direction, (2) the fixation was not within 40pixel of the central fixation dot at trial onset, (3) if the saccade was faster than $100 \mathrm{msec}$ and therefore probably not target-related, (4) if the saccade was slower than $5 \mathrm{sec}$ or (5) if too many large changes in gaze position ( $>20 \%$ of samples differing from the previous sample by more than 20 pixel/ $0.2^{\circ}$ ) occurred in the data, probably related to noise. For the purpose of EEG analysis, all trials with particularly fast saccades (latency $<140 \mathrm{msec}$ ) were excluded to avoid confoundation of the data. Note that Kulke (2015) originally used a $180 \mathrm{msec}$ criterion, as the saccades were on average slower in their study.

\section{EEG data processing}

EEG data processing was conducted in Matlab (version 2017a), using the EEGLAB toolbox. For all participants, external channels removed, the data was resampled to $500 \mathrm{~Hz}$ (the same sampling rate as the eye-tracking data, allowing for extraction of the pre-refined time-windows of previous studies which are reported in full $\mathrm{ms}$ ), Butterworth filters with a $2^{\text {nd }}$ order highpass frequency of $0.01 \mathrm{~Hz}$, and a $2^{\text {nd }}$ order low-pass of $25 \mathrm{~Hz}$ were applied, line noise at $50 \mathrm{~Hz}$ and the respective harmonics was removed using the CleanLine function. The data was re-referenced to the average reference. The eye-tracking data was used to exclude trials with early or incorrect saccades and noisy eye-tracking data. Note that two different time windows were extracted. In order to analyze the $P 1$, which occurs before eye-movements, the data was epoched -0.2 to $0.18 \mathrm{sec}$ around target onset and a $200 \mathrm{msec}$ baseline was subtracted. This short epoch was chosen, as it should be unaffected by eye-movements. Artefactual trials with the maximum voltage larger than $+/-100 \mu \mathrm{V}$, a trend of 50 or a high improbability were rejected. For each condition, the maximum P1 amplitude and its peak latency was determined $100-180 \mathrm{~ms}$ after stimulus onset in a left $(\mathrm{O} 1, \mathrm{PO} 7, \mathrm{PO} 3)$ and right $(\mathrm{O} 2, \mathrm{PO} 4, \mathrm{PO})$ occipital cluster, based on previous research (Kulke et al., 2016a).

In order to investigate later responses (EPN and $L P C$ ), a longer time window was extracted and a slightly different analytic approach was chosen, as these responses may be affected by eyemovements: To exclude noise due to eyemovements, an independent component analysis (ICA) was conducted. For this purpose, a filter with a frequency of $1 \mathrm{~Hz}$ was applied to a copy of the dataset. The resulting data was then epoched into epochs of $[-0.2 ; 1] \mathrm{s}$ around target onset (note that this epoch differs from the time window extracted for the P1 analysis, which is unaffected by eye-movements). An ICA was conducted using the runica command of the EEGLAB package. The ICA weights from the strongly filtered dataset were applied to the original dataset. Two trained coders independently judged the Independent Components (ICs) for each participant. Those artefactual ICs that overlapped between both coders were excluded from further analysis (number of excluded ICs: $M=2.8, S D=1.22, \min =1$, $\max =6$ ).

The mean EPN amplitude was extracted between 250 and $300 \mathrm{~ms}$ after stimulus onset in an occipito-parietal electrode cluster averaged across electrodes O1, O2, P9, P10, PO7 and PO8. The mean LPC amplitude was quantified in a time window between 400 and $600 \mathrm{~ms}$ after stimulus onset in an occipito-parietal electrode cluster averaged across $\mathrm{Pz}, \mathrm{POz}$, 
PO3 and PO4. Regions of interest and time windows for both components were based on previous research (Kulke, Bayer, Grimm, \& Schacht, 2018; Werheid, Schacht, \& Sommer, 2007).

\section{Statistical analyses}

Analyses were conducted as preregistered with the Open Science Framework unless otherwise notes. For simplification, two-tailed p-values are reported below; however, significance decisions would be identical for one-tailed p-values, which were pre-registered. As the tests were pre-registered to directly test previously specified hypotheses, no additional corrections of $\mathrm{p}$-values were applied. Repeated measures Analyses of Variance (ANOVA) were conducted using the aov function and t-tests using the t.test function in $\mathrm{R}$ (Core Team, 2012). Effect sizes were computed as Cohen's d for t-tests using the cohen.d() command from the effsize library and as partial eta squared for ANOVAs, using the EtaSq() command from the DescTools library in R. Note that in addition to the preregistered analyses, Bayes factors were computed to investigate whether the Null hypothesis or the alternative hypothesis are more likely, using the BayesFactor package in R (Morey \& Rouder, 2015) with the respective commands ttestBF and anovaBF. Effects of personality traits were investigated with linear models, using the $\mathrm{lm}$ function in $\mathrm{R}$. Bayes factors were computed using the lmBF function. Shapiro-Wilk tests for normality showed that all outcome measures were normally distributed in all conditions (all $\mathrm{p}>.1$ ). Residual plots showed equal variances for all ANOVA.

\section{Results - preregistered sample}

In this section, results are reported for the originally pre-registered dataset of 24 participants.

\section{Saccade latency}

The overall average saccade latency was $188.2 \mathrm{~ms}(S D=24.4 \mathrm{~ms}, \min =141.8 \mathrm{~ms}$, $\max =241.9 \mathrm{~ms})$. A repeated measures
ANOVA showed no effect of valence (happy, neutral, angry) on saccade latency, $F(2,46)=0.847, p=.435, \eta^{2}$ part $=0.036$, $B F_{10}=0.219$. T-tests showed no difference in saccade latency between happy $(M=$ $0.188, S D=0.025,95 \% C I=[0.177,0.198])$ vs neutral $(M=0.189, S D=0.026,95 \% C I$ $=[0.178,0.200])$ faces, $t(23)=-1.56, p=$ $.132, d=-0.058, B F_{10}=0.621$, angry $(M=$ $0.188, S D=0.024,95 \% C I=[0.178,0.198])$ vs neutral faces, $t(23)=-0.81, p=.428, d=$ $-0.045, B F_{10}=0.288$, or between happy vs angry faces, $t(23)=-0.31, p=.763, d=$ $0.015, B F_{10}=0.224$.

\section{Early posterior response (P1)}

A repeated measures ANOVA showed no effect of valence on the latency of the early posterior response, $F(2,46)=0.149, p=$ $.862, \eta_{\text {part }}^{2}=0.006, B F_{10}=0.130$. T-tests showed no difference in latency between happy $(M=131.77, S D=13.47,95 \% C I=$ [126.08, 137.46]) vs neutral $(M=132.29$, $S D=12.09,95 \% C I=[127.19,137.40])$, $t(23)=-0.324, p=.749, d=-0.041, B F_{10}=$ 0.225 , angry $(M=131.44, S D=13.29$, $95 \% C I=[125.83,137.05])$ vs neutral, $t(23)$ $=-0.476, p=.639, d=-0.067, B F_{10}=0.238$, and happy vs angry faces, $t(23)=0.259, p=$ $.798, d=0.025, B F_{10}=0.221$.

A repeated measures ANOVA showed no effect of valence on the amplitude of the early posterior response, $F(2,46)=0.469, p=.628, \eta^{2}$ part $=0.020$, $B F_{10}=0.167$ (Figure 2). T-tests showed no difference in amplitude between the happy $(M=4.32, S D=2.47,95 \% C I=[3.28,5.36])$ vs neutral $(M=4.19, S D=2.41,95 \% C I=$ $[3.18,5.21]), t(23)=1.04, p=.311, d=$ $0.052, B F_{10}=0.347$, angry $(M=4.23, S D=$ $2.27,95 \% C I=[3.27,5.18])$ vs neutral, $t(23)$ $=0.19, p=.848, d=0.013, B F_{10}=0.218$, and happy vs angry faces, $t(23)=0.77, p=$ $.447, d=0.041, B F_{10}=0.281$.

\section{EPN}

After eye-movement components had been removed using ICA, a repeated measures ANOVA showed a significant effect of valence on the amplitude of the EPN amplitude, $F(2,46)=7.550, p=.001, \eta^{2}$ part 
$=0.247, B F_{10}=22.048$ (Figure 3). Followup t-tests showed a significant difference in amplitude between the angry $(M=2.15, S D$ $=2.86,95 \% C I=[0.94,3.36])$ vs neutral condition $(M=2.75, S D=2.76,95 \% C I=$ $[1.58,3.91]), t(23)=-5.07, p<.001, B F_{10}=$
627.313, $d=-0.212$, between happy $(M=$ $2.22, S D=2.92,95 \% C I=[0.99,3.46])$ vs neutral, $t(23)=-2.60, p=.016, d=-0.184$, $B F_{10}=3.293$, but not between happy vs angry, $t(23)=0.43, p=.674, d=0.026$, $B F_{10}=0.233$.

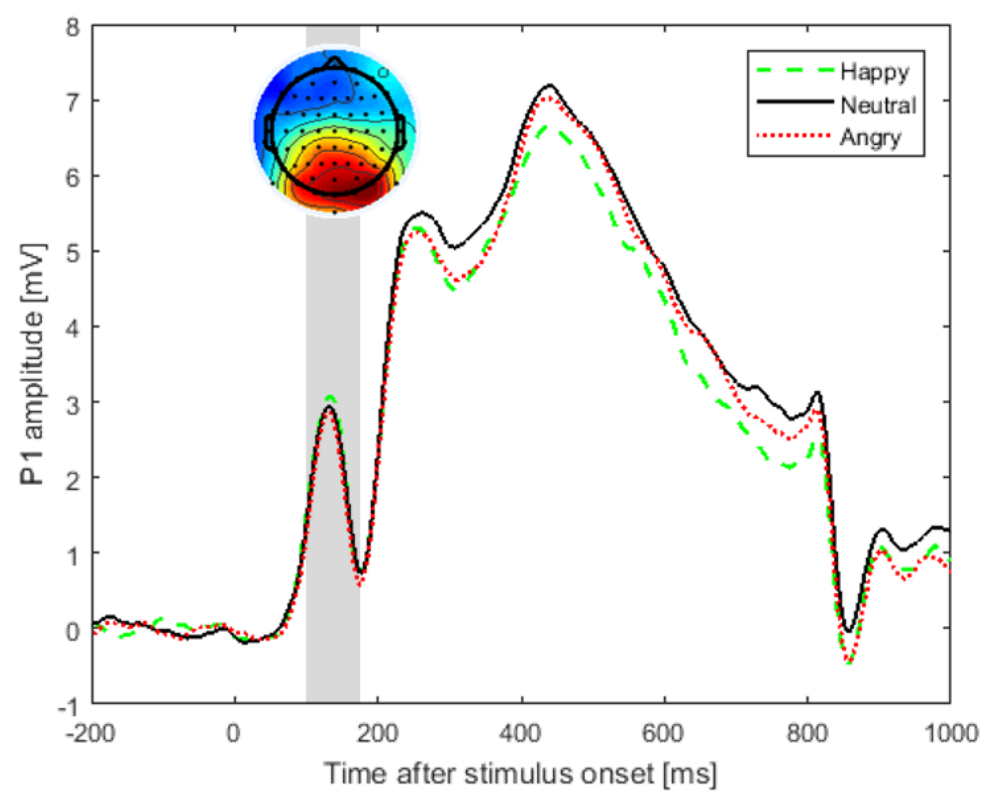

Figure 2. Wave plots, averaged across the left and the right cluster, and topographical distributions of the P1. Happy, neutral and angry face conditions do not differ significantly.

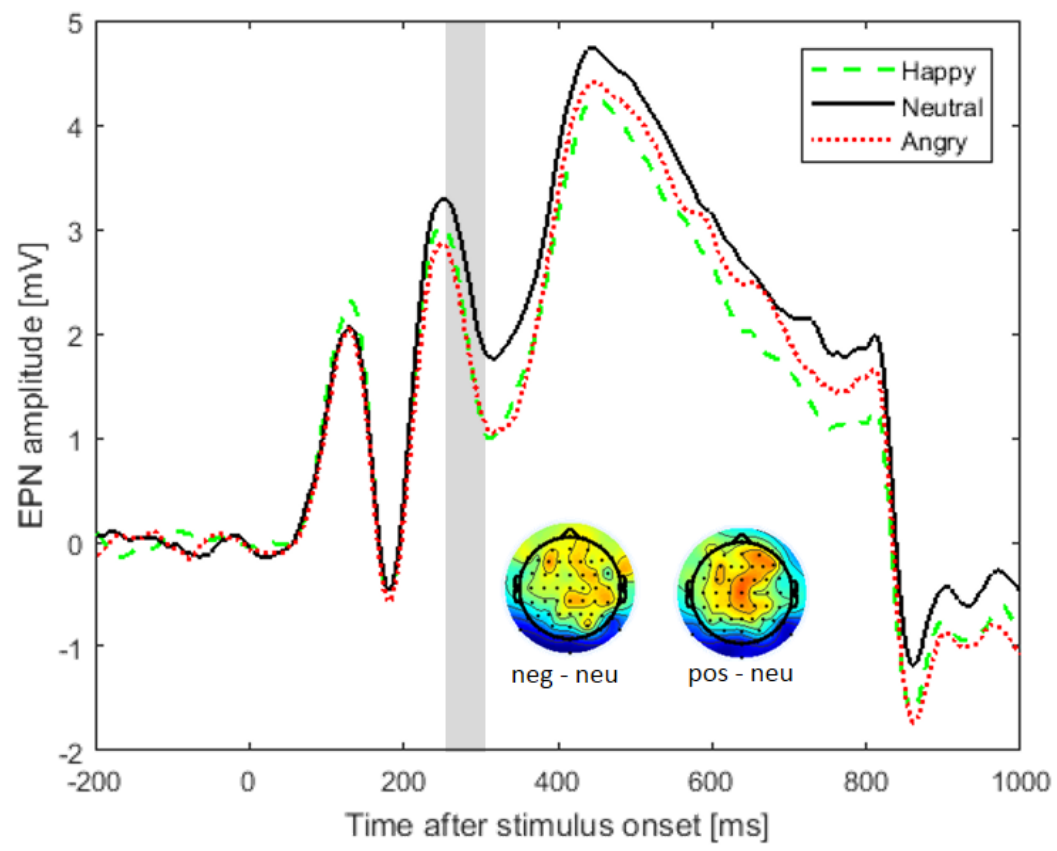

Figure 3. Wave plots of the EPN. Topographical plots are displayed for the difference of negative minus neutral and positive minus neutral.

\section{LPC}

After eye-movement components had been removed using ICA, a repeated measures
ANOVA showed no effect of valence on the amplitude of the LPC, $F(2,46)=1.842, p=$ $.170, \eta_{\text {part }}^{2}=0.074, B F_{10}=0.456$ (Figure 4). 
T-tests showed no significant difference in amplitude between the angry $(M=6.26, S D$ $=2.44,95 \% C I=[5.23,7.28])$ vs neutral condition $(M=6.14, S D=2.23,95 \% C I=$ $[5.20,7.08]), t(23)=0.68, p=.503, B F_{10}=$
$0.265, d=0.048$, between happy $(M=5.91$, $S D=2.55,95 \% C I=[4.83,6.98])$ vs neutral, $t(23)=-1.55, p=.134, B F_{10}=0.616, d=-$ 0.100 , and happy vs angry, $t(23)=-1.51, p$ $=.144, B F_{10}=0.582, d=-0.141$.

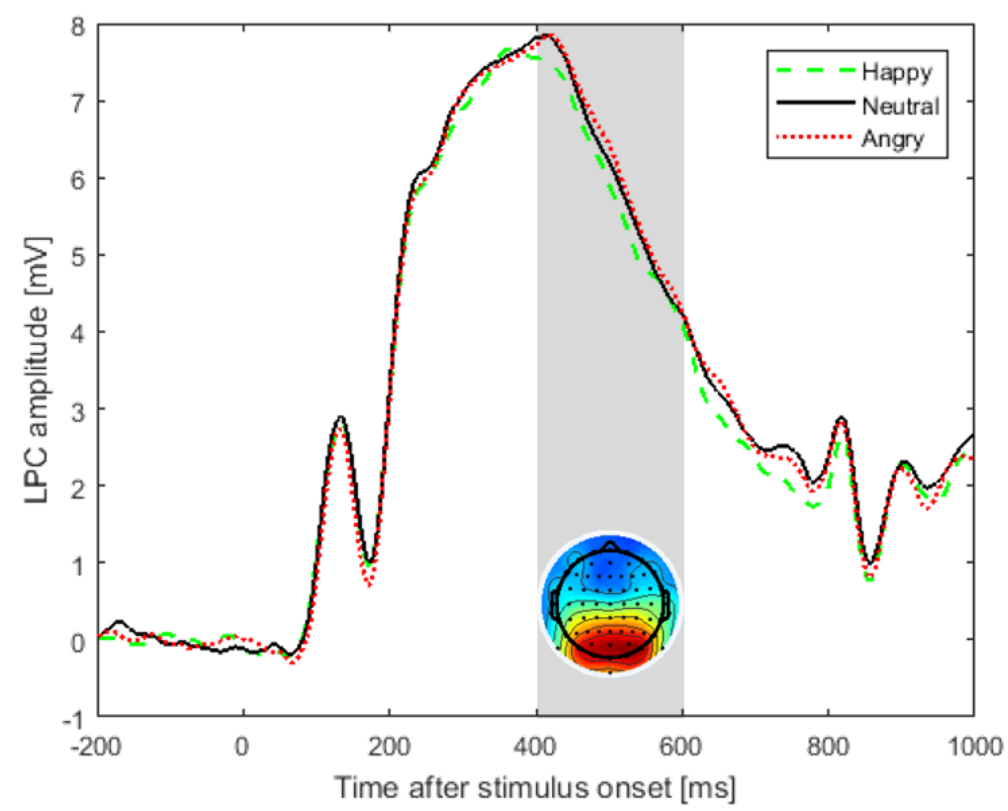

Figure 4. Wave plots and topographical distributions of the LPC. Happy, neutral and angry face conditions do not differ significantly.

\section{Personality traits}

To test if people with high compared to low BIS scores shift faster to angry faces, a linear model was used to predict saccade latency and P1 latency in the angry face condition from BIS score. It showed no significant effect of BIS on saccade latency, $F(1,22)=0.104, p=.750, B F_{10}=0.388$, or on P1 latency, $F(1,22)=0.762, p=.392$, $B F_{10}=0.492$.

In order to test if people with high compared to low BAS scores shift faster to happy faces, a linear model was used to predict saccade and P1 latency from BAS score, showing no significant effect of BAS score on saccade latency, $F(1,22)=0.092$, $p=.764, B F_{10}=0.386$, or on P1 latency, $F(1,22)=0.054, p=.818, B F_{10}=0.381$.

Analyses for the Reading Mind in the Eyes test were pre-registered conditional on significant effects of previous analyses, therefore, only the EPN was further investigated. It showed no effect of RME score, $F(1,22)=2.307, p=.143$, $B F_{10}=0.980$, and no interaction, $F(2,44)=$
$0.414, p=.664, B F_{10}=0.126$, but the effect of condition was confirmed, $F(2,44)=$ $7.358, p=.002, B F_{10}=23.740$.

\section{Results - Analyses of larger sample}

The following analyses were conducted on the full sample of 34 participants.

\section{Saccade latency}

The overall average saccade latency was $186.9 \mathrm{~ms}(S D=25.2 \mathrm{~ms}, \min =141.8 \mathrm{~ms}$, $\max =241.9 \mathrm{~ms})$. A repeated measures ANOVA showed no effect of valence (happy, neutral, angry) on saccade latency, $F(2,66)=1.977, p=.147, \eta^{2}$ part $=0.057$, $B F_{10}=0.423$. T-tests showed a difference in saccade latency between happy $(M=0.186$, $S D=0.025,95 \% C I=[0.177,0.195])$ vs neutral $(M=0.188, S D=0.026,95 \% C I=$ $[0.179,0.197])$ faces, $t(33)=-2.30, \mathrm{p}=$ $.028, d=-0.077, B F_{10}=1.828$, but not angry $(M=0.187, S D=0.025,95 \% \mathrm{CI}=[0.178$, $0.196])$ vs neutral faces, $t(33)=-0.58, p=$ $.566, d=-0.028, B F_{10}=0.215$, or between 
happy vs angry faces, $t(23)=-1.388, p=$ $.174, d=-0.051, B F_{10}=0.441$.

\section{Early posterior response (P1)}

A repeated measures ANOVA showed no effect of valence (happy, neutral, angry) on the latency of the early posterior response, $F(2,66)=0.304, p=.739, \eta^{2}$ part $=0.009$, $B F_{10}=0.114$. T-tests showed no difference in latency between happy $(M=131.09, S D$ $=13.01,95 \% C I=[126.55,135.63]) \mathrm{vs}$ neutral $(M=130.19, S D=12.07,95 \% C I=$ $[125.98,134.40]), t(33)=0.523, p=.604, d$ $=0.071, B F_{10}=0.209$, angry $(M=129.84$, $S D=13.08,95 \% C I=[125.27,134.40]) \mathrm{vs}$ neutral, $t(33)=-0.183, p=.856, d=-0.028$, $B F_{10}=0.187$, and happy vs angry faces, $t(33)=1.010, p=.320, d=0.096, B F_{10}=$ 0.294 .

A repeated measures ANOVA showed no effect of valence (happy, neutral, angry) on the amplitude of the early posterior response, $F(2,66)=0.666, p=$ $.517, \eta^{2}$ part $=0.020, B F_{10}=0.153$. T-tests showed no difference in amplitude between the happy $(M=4.12, S D=2.37,95 \% C I=$ $[3.29,4.94])$ vs neutral $(M=4.02, S D=$ $2.32,95 \% C I=[3.21,4.83]), t(33)=0.88, p$ $=.386, d=0.041, B F_{10}=0.262$, angry $(M=$ $3.99, S D=2.27,95 \% C I=[3.20,4.78])$ vs neutral, $t(33)=-0.21, p=.836, d=-0.012$, $B F_{10}=0.188$, and happy vs angry faces, $t(33)=1.29, p=.206, d=0.053, B F_{10}=$ 0.392 .

\section{EPN}

After removing eye-movement related components with ICA, a repeated measures ANOVA showed a significant effect of valence (happy, neutral, angry) on the EPN amplitude, $F(2,66)=13.08, p<.001, \eta^{2}$ part $=0.284, B F_{10}=1140.945$. T-tests showed a significant difference in amplitude between the happy $(M=1.95, S D=2.84,95 \% C I=$ $[0.96,2.94])$ vs neutral $(M=2.49, S D=$ $2.77,95 \% C I=[1.52,3.45]), t(33)=-3.37$, $p=.002, B F_{10}=18.024, d=-0.190$, angry $(M=1.85, S D=2.76,95 \% C I=[0.89,2.81])$ vs neutral, $t(33)=-6.54, p<.001, B F_{10}=$ $78722.45, d=-0.230$, but not between happy vs angry faces, $t(33)=0.75, p=.458$, $B F_{10}=0.239, d=0.037$.

\section{LPC}

After removing eye-movement related components with ICA, a repeated measures ANOVA showed a marginal effect of valence (happy, neutral, angry) on the LPC amplitude, $F(2,66)=3.168, p=.049, \eta^{2}$ part $=0.088, B F_{10}=1.084$. T-tests showed no significant difference in amplitude between the happy $(M=5.86, S D=2.55,95 \% C I=$ $[4.97,6.75])$ vs neutral $(M=6.10, S D=$ $2.34,95 \% C I=[5.28,6.92]), t(33)=-1.97$, $p=.058, d=-0.097, B F_{10}=1.018$, angry $(M$ $=6.22, S D=2.51,95 \% C I=[5.34,7.09]) \mathrm{vs}$ neutral, $t(33)=0.92, p=.364, d=0.050$, $B F_{10}=0.272$, or between the happy vs angry condition, $t(33)=-2.04$, but note $p=$ $.050, d=-0.142, B F_{10}=1.144$.

\section{Personality traits}

To test if people with high compared to low BIS scores shift faster to angry faces, a linear model was used to predict saccade latency and P1 latency in the angry face condition from BIS score, showing no significant effect of BIS on saccade latency, $F(1,32)=1.055, p=.312, B F_{10}=0.494$, or on P1 latency, $F(1,32)=2.198, p=.148$, $B F_{10}=0.760$.

In order to test if people with high compared to low BAS scores shift faster to happy faces, a linear model was used to predict saccade and P1 latency from BAS score, showing no significant effect of BAS score on saccade latency, $F(1,32)=1.697$, $p=.202, B F_{10}=0.630$, or on P1 latency, $F(1,32)=0.430, p=.517, B F_{10}=0.388$.

\section{RME Score}

Saccade latency showed no effect of RME score, $F(1,32)=2.44, p=.128, B F_{10}=$ 0.758 , condition, $F(2,64)=1.986, p=.146$, $B F_{10}=0.330$, and no interaction, $F(2,64)=$ $1.135, p=.328, B F_{10}=0.205$. P1 latency and P1 amplitude showed no main or interaction effects of RME score.

After ICA EPN showed no effect of RME score, $F(1,32)=1.621, p=.212, B F_{10}$ $=0.826$, and no interaction, $F(2,64)=$ 
$0.373, p=.690, B F_{10}=0.105$, but confirmed the effect of condition, $F(2,64)=12.833, p$ $<.001, B F_{10}=1105.639$. After ICA LPC showed no significant main effect of RME score or interaction with condition, but confirmed the marginal effect of condition, $\mathrm{F}(2,64)=3.191, \mathrm{p}=.048, B F_{10}=1.368$.

\section{samples \\ Summary of differences between}

In summary, all effects found for 34 participants were comparable with the effects found for 24 participants, with the only difference being that for 34 , but not for 24 participants, saccades were significantly faster in the happy $(M=0.186, S D=0.025$, $95 \% C I=[0.177,0.195])$ compared to the neutral $(M=0.188, S D=0.026,95 \% C I=$ $[0.179,0.197])$ condition, $t(33)=-2.30, p=$ .028 , and that a marginal valence effect now occurred on the LPC that was not confirmed in follow-up t-tests.

\section{positions}

\section{Exploratory analysis of fixation}

Due to a reviewer suggestion, fixation locations were compared in a nonpreregistered exploratory analysis between conditions to exclude the possibility that any of the observed EPN effects may be related to the fixation pattern. For this purpose, the first fixation was determined as the gaze location at the time when the first saccade was completed (i.e. a change in gaze position between two subsequent samples larger than 20 pixel/ $0.2^{\circ}$ was no longer detected). These gaze positions were compared in the full sample of 34 participants.

Dependent samples t-tests showed no significant differences between conditions in $\mathrm{x}$-coordinates of the fixation location for stimuli presented on the left side of the screen (happy vs. neutral: $t(33)=$ $-1.79, p=.082, d=-0.058$; happy vs. angry: $t(33)=-0.52, p=.604, d=-0.024$; neutral vs. angry: $t(33)=0.95, p=.348, d=0.034)$ or for stimuli presented on the right side of the screen (happy vs. neutral: $t(33)=0.55$, $p=.585, d=0.024$; happy vs. angry: $t(33)$
$=1.10, p=.280, d=0.055$; neutral vs. angry: $t(33)=0.60, p=.554, d=0.032$ ).

There were no significant differences between conditions in $y$ coordinates of the fixation location for stimuli presented on the left side (happy vs. neutral: $t(33)=0.58, p=.563, d=0.022$; happy vs. angry: $t(33)=0.89, p=.383, d=$ 0.047 ; neutral vs. angry: $t(33)=0.77, p=$ $.448, d=0.025)$. Participants fixated slightly higher (measured through $\mathrm{y}$ coordinates) when happy faces compared to neutral or angry faces were presented on the right side of the screen (happy vs. neutral: $t(33)=2.42, p=.021, d=0.125$, mean difference $=2.2$ pixel; happy vs. angry: $t(33)=2.54, p=.016, d=0.122$, mean difference $=2.2$ pixel; neutral vs. angry: $t(33)=0.01, p=.991, d=0.001)$, but note that this difference has a negligible effect size and would not withstand Bonferroni correction for multiple comparisons. Furthermore, the difference between conditions of 2.2 pixel $(0.02 \mathrm{~cm})$ is equivalent to only $0.4 \%$ of the size of the target stimulus, confirming that this should only have a negligible effect.

In summary, there were only negligible differences in fixation location between conditions. In particular, no differences were observed in the $\mathrm{x}$ dimension. This dimension is crucial as refixations occur from the center to the left or right periphery. Therefore, the fixation position should not have affected any observed effects.

\section{Exploratory analysis of lateralization of the P1 \\ An additional non-preregistered} exploratory analysis was conducted to investigate whether the lateralization of the P1 due to the peripheral presentation of stimuli may interact with valence effects. An ANOVA was conducted to predict P1 latency from Valence and the Brain hemisphere (ipsi- or contralateral to the target), showing no effect of Valence, $F(2$, $66)=0.31, p=.732, \eta_{\text {part }}^{2}=0.009$, Brain hemisphere, $F(1,33)=3.23, p=.081, \eta^{2}$ part $=0.089$, and no interaction, $F(2,66)=0.31$, 
$p=.734, \eta_{\text {part }}^{2}=0.009$. An ANOVA predicting P1 amplitude from Valence and Brain hemisphere (ipsi- or contralateral to the target) showed no effect of Valence, $F(2,66)=2.44, p=.095, \eta^{2}$ part $=0.069$, and no interaction, $F(2,66)=0.51, p=.603$, $\eta^{2}$ part $=0.015$, but a significant effect of Brain hemisphere, $F(1,33)=21.52, p<$ $.001, \eta^{2}$ part $=0.395$, with larger amplitudes in the ipsilateral $(M=4.63, S D=2.15$, $95 \% C I=[4.12,5.13])$ than in the contralateral $(M=3.82, S D=2.23,95 \% C I$ $=[3.29,4.34])$ hemisphere. In summary, the amplitudes were lateralized, in line with previous research (Kulke et al., 2016a); however, adding the lateralization as a factor to the analysis did not change the lack of valence effects.

\section{Discussion}

The current study combined eye-tracking and EEG to measure overt attention shifts towards neutral and emotional faces.

Regarding latencies, there were no effects of emotional expression on the latency of saccades or neural responses. Note, however, that there was a tendency for saccades and posterior responses to be faster towards emotional than neutral faces. When additional participants were tested, the saccades towards happy faces were confirmed to be significantly faster than towards neutral faces, in line with previous findings by Calvo and Nummenmaa (2008), but no difference between emotional and neutral expressions in general occurred, contrasting the suggestion by Johnson Jr (1988), but in line with Hunt et al. (2007). No emotion effects were visible for neural response latencies, in line with findings by Batty and Taylor (2003) regarding emotional salience effects, but contrasting findings regarding visual salience effects (Kulke et al., 2016a; Kulke, Atkinson, \& Braddick, under revision). This contrasts the initial hypothesis that saccade latencies and early posterior ERP latencies differ between emotional and neutral faces. Kulke et al. (under revision) found saccades to be slower when attention was shared between a physically salient distractor and a target, i.e. less attention was directed towards a peripheral target than when full attention was directed towards the peripheral target (Kulke, 2015). The current results suggest that emotional content does not affect eyemovements as salient distractors do.

Regarding early ERP amplitudes, no effects of emotional content were observed on P1 amplitude even after additional participants were tested. Bayesian analyses confirm the absence of such effects. This is in line with previous work showing the P1 amplitude to be unaffected by emotional content (Leppänen et al., 2007; Schupp et al., 2003; Zhang et al., 2012) and suggests that $\mathrm{P} 1$ responses are similar when attention can be shifted overtly to when fixation is maintained.

In contrast, emotional content did affect later responses. The EPN was significantly more negative for emotional than neutral faces, as predicted in the hypothesis. This is in line with previous studies finding emotion effects on the EPN (e.g. Hammerschmidt et al., 2018; Schupp et al., 2003) and confirms that this component is a reliable indicator of emotional content and its attentional draw. Notably, the EPN time windows $(250-300 \mathrm{~ms})$ might overlap with the time point during which saccades occur. As eye components were removed using ICA, the interference was minimized and as the EPN is measured in posterior electrode sites, the effect of eye movement artifacts should be minimal. Furthermore, in the current study most saccades occurred between 180-200ms and should therefore be completed before the EPN time-window. As eye movement latencies or fixation locations did not differ between conditions, the eye-movements are unlikely to differentially affect the ERPs depending on emotional expression. However, it should be noted that the visual input changes during the eye movement and that those responses occurring after refixation may be affected by the central (rather than peripheral) fixation of the faces. As the EPN is measured less than $100 \mathrm{~ms}$ after the refixation, it is unlikely to be affected by the refixation as responses would usually occur 
later. Therefore, the current study shows that EPN modulations can be observed not only during fixation but also when participants can move their eyes freely.

In contrast to our hypothesis, the LPC amplitude did not significantly differ between conditions in the original sample and only showed marginal differences when more participants were included. This contrasts some previous findings (Bublatzky et al., 2014; Eimer \& Holmes, 2002; Recio et al., 2011; Schacht \& Sommer, 2009; Schupp et al., 2004). In line with some other recent findings (Ashley, Vuilleumier, \& Swick, 2004; Kulke et al., 2018), our results suggest that the LPC may not be a reliable marker of emotional content. In the current study, facial expressions were not task-relevant, as participants moved their eyes in each trial. As the LPC is task-dependent (Picton, 1992), it may only show effects if the expression is relevant to the participants' task. Furthermore, the LPC has been suggested to reflect deeper processing of stimuli (Schupp et al., 2006) or mental imagery (Lee et al., 2010). No deeper processing was required in the current task, as participants only needed to make eyemovements but no cognitive judgements about the images, which may have led to the lack of LPC effects. It must be noted that the electrode cluster used for the LPC may be affected by eye-movement artifacts. Due to the ICA and the selected time window (400$600 \mathrm{~ms}$, after saccade have occurred), the artefacts should however only have a negligible effect on the reported findings.

No modulations of the effects occurred based on BIS/ BAS scores. This may either suggest that overt attention shifts to emotional faces are unaffected by these personality traits, or that larger sample sizes are required to detect such between-subjects effects. The Bayes factors suggest that it is more likely that there is no effect of these personality traits (2.0 to 2.6 times more likely) than that there is one. This finding contrasts the original hypotheses that people with high BIS scores would react faster to angry faces and people with high
BAS scores would react faster to happy faces. It may be that BIS and BAS scores reflect higher level processing of explicit motives and are therefore unrelated to the simple reflexive visual processing of emotions measured in the current study. Early processing, reflected in P1 and saccadic responses may be directed based on physical salience rather than emotional salience. Furthermore, there were no effects of Reading Mind in the Eye test scores on EPN amplitude; however, the Bayes factors remain inconclusive. Further research is required to investigate whether the ability to recognize complex emotions is related to fast attention shifts to emotional faces.

In summary, although early P1 responses and saccade latencies were unaffected by emotional expressions, the later EPN component showed clear valence effects. This is interesting, as in the current study, neural emotion effects only occurred after eye-movements. Although eyemovement latencies were not affected by emotional content in the current study, previous research found them to be modulated by visual salience (Kulke, 2015; Kulke et al., 2016a, under revision), particularly for fast saccades occurring directly after the onset of a salient stimulus (Donk \& van Zoest, 2008). Specifically, eye-movement latencies and P1 latencies were slower when two salient targets were competing for attention than when one single target was visible (Kulke et al., under revision). In future it would be interesting to investigate interactions of competition and emotional valence. Previous research suggests that early $\mathrm{C} 1$ responses are enlarged towards fearful faces when attention is shifted to them covertly (Pourtois et al., 2004); however, no such effects have been investigated in overt attention shift tasks. It remains to be investigated whether faster or enhanced responses may occur towards emotional faces that are competing with neutral ones.

Taken together, the findings suggest that the first attentional draw may be based on visual salience of the peripheral occurrence (Donk \& van Zoest, 2008; 
Kulke et al., 2016a, under revision), while only later neural processing is affected by emotional content with enhanced amplitudes for emotional stimuli. It has previously been suggested that emotional expressions of faces are only differentially processed when sufficient attentional resources are available, suggesting the involvement of top-down processes (Pessoa, McKenna, Gutierrez, \& Ungerleider, 2002). The current study shows that attentional responses (eyemovements) occur before neural response modulations, in line with the requirement of attention before emotion processing. Therefore, physical salience may promptly shift attention in a bottom-up manner, while emotional content requires additional processing. Previous behavioural findings from manual responses and eye-movements in a top-down attention task suggest that effects of emotional expressions on eyemovements may be related to the visual salience of happy facial expressions (Calvo \& Nummenmaa, 2011). Similarly, the study by Bublatzky et al. (2014), which showed P1 effects of emotional expression, did not control stimulus luminance which may have affected findings. As the current study controlled luminance, differences in visual salience were kept to a minimum. Therefore, effects of emotional rather than physical salience could be measured.

It must be noted that EEG does not measure subcortical processes, which play a crucial role during eye-movements. For example, the superior colliculus is involved in the generation of eye-movements (e.g. Hanes \& Wurtz, 2001; Neggers, Raemaekers, Lampmann, Postma, \& Ramsey, 2005; review of primate literature: Wurtz \& Albano, 1980) and also responds to visual stimuli (Goldberg \& Wurtz, 1972; Schiller \& Stryker, 1972) and therefore plays a role during attention shifting (Neggers et al., 2005). It is possible that early emotion modulations do occur but on a subcortical level. Future MRI or MEG studies could shed light in this question.
One difference between the current study and other studies demonstrating emotion effects is that the emotional expression of faces was not task-relevant in the current study. Participants made eyemovements independent of the facial expression. The study therefore only required bottom-up processing of emotional expression but involved no top-down component, allowing to extract bottom-up effects more clearly. It is possible that participants suppressed emotional processing as it was not task-relevant. This would speak against the view of automatic processing of emotions (Öhman, 2002; Pourtois et al., 2004) and instead suggest that attention is required for processing of emotional expressions (for an overview, see Pessoa \& Ungerleider, 2005). Emotion effects on saccade latencies and early ERPs may be task-dependent and therefore not occurring in a purely bottom-up task. Alternatively, overtness of attention shifts may play a role. Participants moved their eyes during attention shifts in the current study, while most previous studies that found P1 effects asked participants to keep their eyes fixated (e.g., Bublatzky et al., 2014; Rellecke et al., 2012). This highlights the importance of studying the more natural overt attention shifts, which may indeed differ from covert shifts.

The current study investigated covert attention shifts to faces with different expression, demonstrating that early neural responses (P1) and saccade latencies are unaffected by emotional content of expressions, as supported by Bayesian statistics, while modulations occur on later neural responses after completion of the eye-movement.

\section{Acknowledgements}

We would like to thank Annekathrin Schacht and Arezoo Pooresmaeili for helpful feedback and Marlene Wessels and Lena Brümmer for help with testing. This project was funded by a Seed Fund of the Leibniz-ScienceCampus Primate Cognition (LSC-SF2017-14). 


\section{References}

Ashley, V., Vuilleumier, P., \& Swick, D. (2004). Time course and specificity of event-related potentials to emotional expressions. Neuroreport, 15(1), 211-216.

Baron-Cohen, S., Wheelwright, S., Skinner, R., Martin, J., \& Clubley, E. (2001). The autismspectrum quotient (AQ): Evidence from asperger syndrome/high-functioning autism, malesand females, scientists and mathematicians. Journal of Autism and Developmental Disorders, 31(1), 5-17.

Baron-Cohen, S., Jolliffe, T., Mortimore, C., \& Robertson, M. (1997). Another advanced test of theory of mind: Evidence from very high functioning adults with autism or Asperger syndrome. Journal of Child psychology and Psychiatry, 38(7), 813-822.

Batty, M., \& Taylor, M. J. (2003). Early processing of the six basic facial emotional expressions. Cognitive Brain Research, 17(3), 613-620.

Bublatzky, F., Gerdes, A., White, A. J., Riemer, M., \& Alpers, G. W. (2014). Social and emotional relevance in face processing: happy faces of future interaction partners enhance the late positive potential. Frontiers in human neuroscience, 8, 493.

Calvo, M. G., \& Nummenmaa, L. (2008). Detection of emotional faces: salient physical features guide effective visual search. Journal of Experimental Psychology: General, 137(3), 471.

Calvo, M. G., \& Nummenmaa, L. (2011). Time course of discrimination between emotional facial expressions: The role of visual saliency. Vision Research, 51(15), 1751-1759.

Carver, C. S., \& White, T. L. (1994). Behavioral inhibition, behavioral activation, and affective responses to impending reward and punishment: the BIS/BAS scales. Journal of personality and social psychology, 67(2), 319.

Corby, J. C., \& Kopell, B. S. (1972). Differential contributions of blinks and vertical eye movements as artifacts in EEG recording. Psychophysiology, 9(6), 640-644.

Core Team, R. (2012). R: A language and environment for statistical computing. Vienna, Austria.

Croft, R., \& Barry, R. (2000). Removal of ocular artifact from the EEG: a review. Neurophysiologie Clinique/Clinical Neurophysiology, 30(1), 5-19.

Dickinson, A., \& Dearing, M. F. (1979). Appetitive-aversive interactions and inhibitory processes. Mechanisms of learning and motivation: A memorial volume to Jerzy Konorski, 203-231.

Donk, M., \& van Zoest, W. (2008). Effects of salience are short-lived. Psychological Science, 19(7), 733-739.

Eimer, M., \& Holmes, A. (2002). An ERP study on the time course of emotional face processing. Neuroreport, 13(4), 427-431.

Faul, F., Erdfelder, E., Lang, A.-G., \& Buchner, A. (2007). G*Power 3: A flexible statistical power analysis program for the social, behavioral, and biomedical sciences. Behavior Research Methods, 39, 175-191.

Foulsham, T., Walker, E., \& Kingstone, A. (2011). The where, what and when of gaze allocation in the lab and the natural environment. Vision Research, 51(17), 1920-1931.

Goldberg, M. E., \& Wurtz, R. H. (1972). Activity of superior colliculus in behaving monkey. I. Visual receptive fields of single neurons. J Neurophysiol, 35(4), 542-559.

Hammerschmidt, W., Kulke, L., Broering, C., \& Schacht, A. (2018). Money or smiles: Independent ERP effects of associated monetary reward and happy faces. PLOS ONE, 13(10), 0206142.

Hanes, D. P., \& Wurtz, R. H. (2001). Interaction of the frontal eye field and superior colliculus for saccade generation. Journal of Neurophysiology, 85(2), 804-815.

Hunt, A. R., Cooper, R. M., Hungr, C., \& Kingstone, A. (2007). The effect of emotional faces on eye movements and attention. Visual Cognition, 15(5), 513-531. doi: $10.1080 / 13506280600843346$ 
Johnson Jr, R. (1988). The amplitude of the P300 component of the event-related potential: Review and synthesis. Advances in psychophysiology, 3, 69-137.

Joyce, C. A., Gorodnitsky, I. F., \& Kutas, M. (2004). Automatic removal of eye movement and blink artifacts from EEG data using blind component separation. Psychophysiology, 41(2), 313-325.

Kulke, L. (2015). Cortical mechanisms of visual attention in typically developing infants and adults. UCL (University College London).

Kulke, L., Atkinson, J., \& Braddick, O. (2016a). Neural differences between covert and overt attention studied using EEG with simultaneous remote eye tracking. Frontiers in Human Neuroscience, 10.

Kulke, L., Atkinson, J., \& Braddick, O. (2016b). Neural mechanisms of attention become more specialised during infancy: Insights from combined eye tracking and EEG. Developmental Psychobiology, 59(2), 250-260.

Kulke, L., Atkinson, J., \& Braddick, O. (under revision). Cortical mechanisms of overt attention shifts: Visual offset prompts the brain to shift attention. Perception.

Kulke, L., Bayer, M., Grimm, A.-M., \& Schacht, A. (2018). Differential effects of learned associations with words and pseudowords on event-related brain potentials. Neuropsychologia. doi: https://doi.org/10.1016/j.neuropsychologia.2018.12.012

Lang, P. J., \& Bradley, M. M. (2010). Emotion and the motivational brain. Biological psychology, 84(3), 437-450.

Lee, K.-Y., Lee, T.-H., Yoon, S.-J., Cho, Y. S., Choi, J.-S., \& Kim, H. T. (2010). Neural correlates of top-down processing in emotion perception: an ERP study of emotional faces in white noise versus noise-alone stimuli. Brain Research, 1337, 56-63.

Leppänen, J. M., Moulson, M. C., Vogel-Farley, V. K., \& Nelson, C. A. (2007). An ERP study of emotional face processing in the adult and infant brain. Child Development, 78(1), 232-245.

Luck, S. J. (2005). An introduction to the event-related potential technique. Cambridge: MIT Press.

Mogg, K., \& Bradley, B. P. (1999). Orienting of attention to threatening facial expressions presented under conditions of restricted awareness. Cognition \& Emotion, 13(6), 713 740.

Morey, R. D., \& Rouder, J. N. (Producer). (2015). BayesFactor: Computation of Bayes Factors for Common Designs. $R$ package version 0.9.12-2. Retrieved from http://CRAN.Rproject.org/package $=$ BayesFactor

Neggers, S., Raemaekers, M., Lampmann, E., Postma, A., \& Ramsey, N. (2005). Cortical and subcortical contributions to saccade latency in the human brain. European Journal of Neuroscience, 21(10), 2853-2863.

Nummenmaa, L., Hyönä, J., \& Calvo, M. G. (2006). Eye movement assessment of selective attentional capture by emotional pictures. Emotion, 6(2), 257.

Nummenmaa, L., Hyönä, J., \& Calvo, M. G. (2009). Emotional scene content drives the saccade generation system reflexively. Journal of Experimental Psychology: Human Perception and Performance, 35(2), 305.

Öhman, A. (2002). Automaticity and the amygdala: Nonconscious responses to emotional faces. Current Directions in Psychological Science, 11(2), 62-66.

Pessoa, L., McKenna, M., Gutierrez, E., \& Ungerleider, L. (2002). Neural processing of emotional faces requires attention. Proceedings of the National Academy of Sciences, 99(17), 11458-11463.

Pessoa, L., \& Ungerleider, L. G. (2005). Visual attention and emotional perception Neurobiology of attention (pp. 160-166): Elsevier.

Picton, T. W. (1992). The P300 wave of the human event-related potential. Journal of clinical neurophysiology, 9(4), 456-479. 
Pivik, R. T., Broughton, R. J., Coppola, R., Davidson, R. J., Fox, N., \& Nuwer, M. R. (1993). Guidelines for the recording and quantitative analysis of electroencephalographic activity in research contexts. Psychophysiology, 30(6), 547-558.

Pourtois, G., Grandjean, D., Sander, D., \& Vuilleumier, P. (2004). Electrophysiological correlates of rapid spatial orienting towards fearful faces. Cerebral Cortex, 14(6), 619633.

Recio, G., Sommer, W., \& Schacht, A. (2011). Electrophysiological correlates of perceiving and evaluating static and dynamic facial emotional expressions. Brain Research, 1376, 66-75.

Rellecke, J., Sommer, W., \& Schacht, A. (2012). Does processing of emotional facial expressions depend on intention? Time-resolved evidence from event-related brain potentials. Biological psychology, 90(1), 23-32.

Saber, G. T., Pestilli, F., \& Curtis, C. E. (2015). Saccade planning evokes topographically specific activity in the dorsal and ventral streams. Journal of Neuroscience, 35(1), 245252.

Schacht, A., \& Sommer, W. (2009). Emotions in word and face processing: early and late cortical responses. Brain and cognition, 69(3), 538-550.

Schiller, P. H., \& Stryker, M. (1972). Single-unit recording and stimulation in superior colliculus of the alert rhesus monkey. Journal of Neurophysiology, 35(6), 915-924.

Schupp, H. T., Flaisch, T., Stockburger, J., \& Junghöfer, M. (2006). Emotion and attention: event-related brain potential studies. Progress in Brain Research, 156, 31-51.

Schupp, H. T., Junghöfer, M., Weike, A. I., \& Hamm, A. O. (2003). Attention and emotion: an ERP analysis of facilitated emotional stimulus processing. Neuroreport, 14(8), 11071110.

Schupp, H. T., Öhman, A., Junghöfer, M., Weike, A. I., Stockburger, J., \& Hamm, A. O. (2004). The facilitated processing of threatening faces: an ERP analysis. Emotion, 4(2), 189.

Strobel, A., Beauducel, A., Debener, S., \& Brocke, B. (2001). Eine deutschsprachige Version des BIS/BAS-Fragebogens von Carver und White. Zeitschrift für Differentielle und diagnostische Psychologie.

Vuilleumier, P. (2005). How brains beware: neural mechanisms of emotional attention. Trends in cognitive sciences, 9(12), 585-594.

Vuilleumier, P., Armony, J. L., Driver, J., \& Dolan, R. J. (2001). Effects of attention and emotion on face processing in the human brain: an event-related fMRI study. Neuron, 30(3), 829-841.

Vuilleumier, P., \& Huang, Y.-M. (2009). Emotional attention: Uncovering the mechanisms of affective biases in perception. Current Directions in Psychological Science, 18(3), 148152.

Werheid, K., Schacht, A., \& Sommer, W. (2007). Facial attractiveness modulates early and late event-related brain potentials. Biological psychology, 76(1-2), 100-108.

Wurtz, R. H., \& Albano, J. E. (1980). Visual-motor function of the primate superior colliculus. Annual review of neuroscience, 3(1), 189-226.

Zhang, D., He, W., Wang, T., Luo, W., Zhu, X., Gu, R., . . Luo, Y.-j. (2014). Three stages of emotional word processing: an ERP study with rapid serial visual presentation. Social cognitive and affective neuroscience, 9(12), 1897-1903.

Zhang, D., Wang, L., Luo, Y., \& Luo, Y. (2012). Individual Differences in Detecting Rapidly Presented Fearful Faces. PLOS ONE, 7(11), e49517. doi: 10.1371/journal.pone.0049517 